\title{
Um ensaio sobre desempenho econômico e participação em cooperativas agropecuárias
}

Sigismundo Bialoskorski Neto*

Resumo: Este ensaio analisa a influência da função objetivo, do número de associados e do desempenho econômico da organização cooperativa na participação de associados nas assembléias gerais ordinárias e em comitês educativos. São analisados dois modelos de regressão que permitem considerar que há uma correlação negativa entre o desempenho econômico e a participação em assembléias gerais, e que a participação em comitês educativos ocorre provavelmente para intensificar as relações sociais em organizações que apresentem problemas de participação econômica do quadro associado. Por fim, também se pode indicar que a participação em empreendimentos coletivos, como as cooperativas, é de fundamental importância para gerar transparência de governança corporativa, e assim há uma dicotomia a ser discutida, pois, quanto melhor é o desempenho econômico das cooperativas, também é menor a participação e, portanto, menos transparente será o processo de gestão e governança, exigindo custos adicionais para suplantar essa deficiência.

Palavras-Chave: cooperativas, participação, governança corporativa.

Classificação JEL: L14, L21, Q13

* Professor do Departamento de Economia da Faculdade de Economia, Administração e Contabilidade de Ribeirão Preto - Universidade de São Paulo. sigbial@usp.br 
Abstract: This essay analyzes the member participation in general assembly and educational committees in agricultural cooperatives in function of economic objective function, members' number, and economic performance. There are analyses of two regression models that show the participation is an inverse function of economic performance in cooperatives and the educational committee participation occurs in functions to intensify the member relations in cooperative with problems with member economic activity. At the end it is possible also indicate that member participation is important to corporate governance transparency in agricultural cooperative but there is a dichotomy because the best economic performance in cooperatives could not incentive member participation and in consequence the governance transparency that needs additional costs to correct this deficiency.

Key words: cooperatives, participation, corporate governance.

JEL Classification: L14, L21, Q13

\section{Introdução}

Quando se discute a questão da cooperação, podem-se fazer análises diferentes sobre este tema e sobre a questão da participação do membro associado nas estruturas de gestão e governança da cooperativa. A primeira, que se torna corrente em alguns países e particularmente no Brasil, é aquela que considera a cooperação como uma forma de voluntarismo e solidariedade por parte dos membros associados, e que teria como base as relações de troca de bens e serviços, muitas vezes até se fazendo uso do escambo, moedas solidárias, ou cupons de troca, tendo-se a participação do associado como algo apenas voluntário (SINGER; SOUZA, 2000; PINHO, 2003).

A segunda analisa a participação econômica dos membros no empreendimento cooperativo em conseqüência da necessidade de crescimento econômico intrínseco destas organizações em função do mercado e da lógica de eficiência explicada e modelada pela economia neoclássica.

Assim, pode-se discutir a participação dos membros associados desde a fase em que as cooperativas se formam como uma importante 
coalizão de interesses em um mesmo grupo étnico, solidário e voluntário, com um forte código de ética, até aquela em que a participação econômica é necessária para o crescimento empresarial impulsionado pelo mercado e pela necessidade de geração de renda e riquezas, internalizando a lógica econômica de maximização de resultados (BIALOSKORSKI NETO, 2004a; SOARES, 1984).

A escola de Munster (ESCHENBURG, 1983) explica que a cooperação deve existir sob parâmetros solidários somente em um primeiro instante destas organizações, em um momento posterior se estabelece um processo em que o sucesso da cooperação somente ocorre se o benefício econômico que o associado encontra sob a forma cooperativada for necessariamente maior que o benefício econômico que este mesmo produtor poderia encontrar de forma autônoma, individual e livre no mercado.

Pode-se discorrer, ainda, sobre algumas características econômicas importantes do processo de participação como os custos de participação e cooperação para cada membro associado. Inicialmente há um custo da perda de parte de sua determinação econômica individual em favor do grupo de interesse da coalizão cooperativa, isto se expressa desde a necessidade de comercialização com regras comuns até o respeito às decisões majoritárias e igualitárias nas estruturas de governança e decisão, que muitas vezes podem ser contrárias aos interesses particulares de determinado associado.

Também há o custo de participação que o regime de cooperação induz para o agente econômico sob o ponto de vista individual, ou seja, há um custo de oportunidade do tempo no processo de participação.

Assim, a intensidade da participação pode ocorrer em proporção inversa aos custos de oportunidade do trabalho e aos custos de oportunidade do tempo do membro associado. Quanto maiores estes custos, menor será a participação, isto é, toda vez em que há um custo de oportunidade significativo do fator de produção trabalho, para o produtor rural, esse não estimulará a participação nas estruturas de governança. Por outro lado, a participação poderá ocorrer, por exemplo, se a importância econômica da decisão a ser tomada na assembléia for maior para o produtor rural que a perda prevista pela ausência em seu trabalho diário na propriedade. 
Mas, ainda há problemas de participação devido ao fato de que as cooperativas apresentam uma estrutura de direitos de propriedade vagamente definidos e poucos incentivos contratuais para a participação (COOK; ILIOPOULOS, 1998; BIALOSKORSKI NETO, 2004b). Deste modo, tanto a cultura organizacional como o ambiente institucional no Brasil podem ter influência na participação dos associados, uma vez que as cooperativas apresentam, em sua maioria, uma função objetivo de distribuição de resultados por meio da prestação de serviços e melhores preços imediatos e, assim, incentivam uma situação de direitos de propriedade vagamente definidos.

O sistema cooperativo agropecuário brasileiro mantém, por característica própria, a não obrigatoriedade contratual nas transações entre os membros e a cooperativa, bem como não há uma obrigação contratual que determine a participação do cooperado e sua cooperativa. Essa característica é interessante para o associado quando se consideram os custos de oportunidade desse associado no mercado, porém pode ser onerosa para a cooperativa, uma vez que pode permitir também a existência de oportunismos contratuais e desvios não interessantes para a eficiência econômica da cooperativa.

É necessário compreender ainda os fatores que possibilitam a manutenção de uma empresa organizada sob a forma coletiva, assim a questão da governança corporativa é importante e, por conseguinte, a participação do membro nas estruturas de governança passa a ser uma variável essencial para garantir a transparência e o sucesso da governança corporativa de uma cooperativa.

O objetivo deste ensaio é discutir os fatores que influenciam a participação dos associados nas cooperativas agropecuárias e, em especial, nas assembléias gerais e comitês educativos. A hipótese inicial é de que o melhor desempenho econômico da cooperativa, ao contrário do que se discute, incentiva a diminuição da participação nas estruturas de governança e, portanto, não incentiva a transparência nas atividades de governança corporativa.

A importância desse tema é que os pressupostos de governança corporativa explanam que as organizações que apresentam melhor resultado econômico são aquelas onde também deve obrigatoriamente haver maior transparência de gestão, e assim há um problema, pois 
as organizações cooperativas caracterizadas pelo melhor desempenho econômico teriam que incentivar também a participação do associado nas suas estruturas de governança.

\section{A participação e o número de cooperados}

Quando se faz referência à participação dos associados em cooperativas agropecuárias, há questões importantes como o número total de membros da organização, visto que o crescimento econômico de uma cooperativa implica freqüentemente em um aumento no número de associados, e isso, por sua vez, pode ser prejudicial para a participação social de cada um dos membros, já que implica em custos.

Esses custos ocorrem provavelmente, entre outros fatores, devido ao fato de que quanto maior é o número de associados também maior será a área geográfica de atuação da cooperativa e provavelmente mais difícil será tanto para o associado participar quanto para a organização cooperativa incentivar essa participação em função do aumento dos custos de oportunidade já descritos.

Esse maior tamanho da cooperativa em termos de associados também implica no fato de que há uma divisão maior nos direitos de decisões nas Assembléias Gerais Ordinárias (AGOs), isto é, como igualitariamente cada membro tem somente um voto, no caso de um maior número de associados este voto não terá mais o mesmo valor relativo quando se compara com uma cooperativa menor e de poucos membros, e isso poderá não incentivar o processo de participação.

Como exemplo, em uma cooperativa com 20 associados, o voto relativo de cada um deles representa 1/20 de poder de decisão, podendo haver uma maior expressão nas assembléias gerais, mas, em uma cooperativa com 1.000 associados, cada voto representa 1/1.000 do poder de decisão e uma probabilidade de menor expressão em uma assembléia geral.

Deste modo, quanto maior o número de associados, há uma tendência de que os custos relativos de oportunidade de participação sejam maiores que os benefícios em decorrência de uma maior divisão relativa de direitos de decisão. O Gráfico 1 mostra a curva descendente dessa relação em função do número de associados. Nesse gráfico é possível perceber que deverá então haver uma maior delegação de poder e direi- 
tos de decisão do associado para os gestores da cooperativa e, com isso, haverá uma modificação nas características e custos das estruturas de governança corporativa.

Gráfico 1. Representação do número de associados em cooperativas, participação, e delegação do poder de decisão - delegação de poder

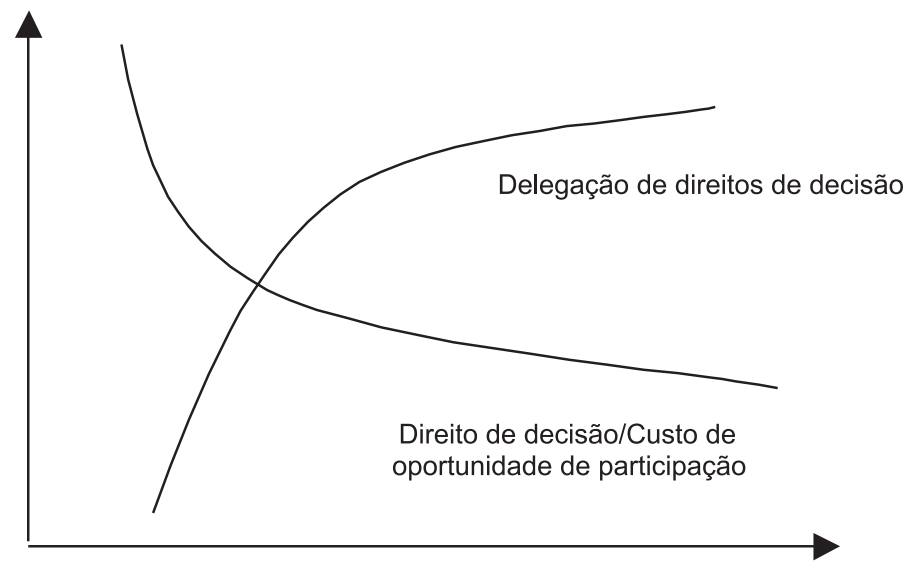

Número de associados

No caso das cooperativas com um maior número de associados, existe também a probabilidade de ocorrer um menor monitoramento entre os membros e, nesse caso, uma menor participação pode criar também um menor compromisso relacional entre a cooperativa e os seus associados; esse processo é conhecido pelo afastamento da gestão da cooperativa do seu quadro social.

Assim, o associado, para participar mais, deverá perceber e obter maiores incentivos e benefícios compensatórios, em função dos custos e benefícios da sua participação. Esses benefícios devem compensar a maior divisão e a conseqüente diminuição de direitos à decisão e poder de voto nas cooperativas com um número muito alto de associados.

O Gráfico 2 mostra a relação empírica entre a participação dos associados em assembléias gerais ordinárias, em proporção, em função do número total de associados de uma cooperativa. Esta relação foi observada em cooperativas agropecuárias do Estado do Paraná em 1999. Neste caso, pode-se analisar que há uma função inversamente propor- 
cional entre participação e número de associados, e que há uma linha de tendência logarítmica sugerindo que a aproximação teórica apresentada no Gráfico 1 pode estar possivelmente correta.

Gráfico 2. Relação entre a participação do associado em Assembléias Gerais Ordinárias e o número de associados em cooperativas agrícolas do Estado do Paraná em 1999. A linha de tendência é uma função logarítmica.

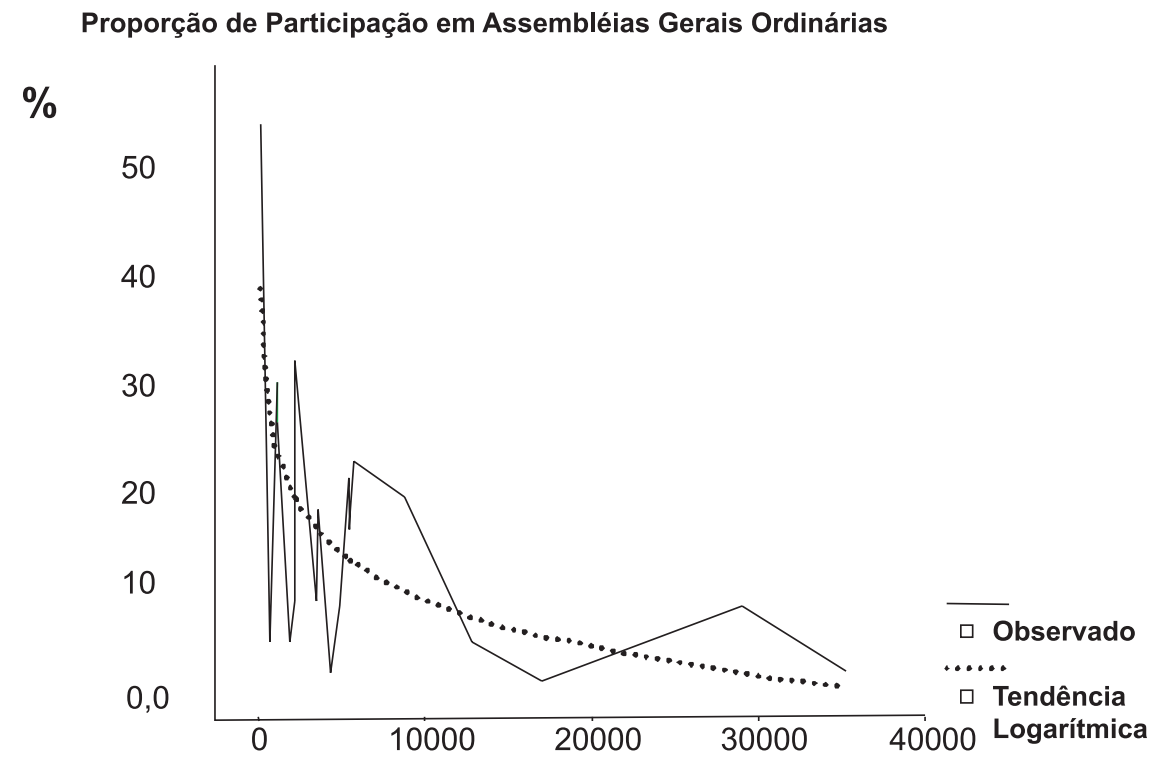

Total de Membros

Fonte: dados de pesquisa

Pode-se aferir então que a participação dos membros em uma cooperativa nas Assembléias Gerais Ordinárias (AGOs) é inversamente proporcional ao número dos associados, não só em função da maior divisão de poder de decisão, como também em função da maior dispersão geográfica do quadro associativo, que pode tornar o custo relativo de oportunidade da participação mais oneroso para o associado. Cooperativas grandes e com muitos associados podem diminuir a intensidade desse problema promovendo assembléias prévias e reuniões preparatórias à AGO em núcleos de produtores. 


\section{A função objetivo da cooperativa e a participação do cooperado}

Uma organização cooperativa pode ter basicamente duas funções objetivo. A primeira é aquela em que o objetivo é distribuir resultados - sobras - aos seus membros, em dinheiro, no final do período contábil; assim é criada uma situação transparente para o membro que pode monitorar o comportamento econômico da organização cooperativa por meio dos valores monetários distribuídos, contudo essa estratégia é pouco comum no Brasil, sendo mais freqüente em outros países.

A segunda estratégia objetiva resultados econômicos e esses resultados são distribuídos aos associados imediatamente em forma de melhores preços de aquisição dos produtos agrícolas ou de venda de insumos, e em forma de prestação de serviços de assistência técnica e outros, o que é muito freqüente no Brasil.

A função objetivo comum no Brasil, de distribuir os resultados da cooperativa em termos de melhores preços imediatos, e serviços como condições de produção e assistência técnica, pode possibilitar uma maior indefinição de direitos de propriedade e de decisões. Isto ocorre uma vez que as relações formais e informais entre os associados beneficiários e os funcionários detentores de direitos residuais de decisão sobre o uso de ativos coletivos e a prestação de serviços passam a ser importantes.

Essa situação pode se constituir em um incentivo ao esforço de participação do membro associado em sua cooperativa, pois trata-se de uma lógica de apropriação de direitos residuais. Desta maneira, se isso ocorrer sem apresentar necessariamente prejuízo perceptível aos demais membros, se constituirá em um incentivo à participação dos membros (BIALOSKORSKI NETO, 2004b).

Essa situação poderia estar intensificando os contratos relacionais e informais entre os membros associados, e assim criando condições de confiança e de imersão social - embeddedness (GRANOVETTER, 1985). As condições de capital social são importantes tanto no ato de formação do empreendimento coletivo como também para que seu crescimento econômico possa estar sustentado em relações sociais.

Todavia, essas relações somente existem se a função objetivo da cooperativa incentivar a distribuição dos resultados por meio de melhores preços e oferta de serviços e, portanto, dependem da função objetivo da cooperativa. 
Pesquisa com presidentes e diretores de cooperativas agropecuárias do Estado de São Paulo mostrou que os cooperados mais relacionados influem mais nas cooperativas, que esses têm também mais vantagens na organização cooperativada e apresentam maior fidelidade (BIALOSKORSKI NETO, 2004b).

\section{Desempenho econômico e participação em cooperativas}

A hipótese inicial deste ensaio é que, se a cooperativa tem um melhor desempenho econômico e financeiro, há uma situação provável de melhores preços e maior oferta de serviços, assim há participação econômica e em transações, mas a participação social do associado nas estruturas de decisão tende a diminuir, uma vez que poderá haver também uma conseqüente e mais intensa prestação de serviços oriunda dos melhores resultados econômicos.

Por outro lado, se a cooperativa não apresenta um bom resultado em seu desempenho econômico, o custo de oportunidade da tomada de decisões se torna maior, bem como há uma tendência de que a oferta em serviços para os membros provavelmente diminua, e assim a participação social nas estruturas de decisão na organização deverá aumentar.

O produtor rural, membro associado à cooperativa, apresenta um perfil de aversão ao risco e apresenta um maior valor marginal para a perda de utilidade do que o valor marginal da utilidade dos benefícios recebidos da cooperativa (HENDRICKSE, 2003). Deste modo, uma vez que a cooperativa apresenta riscos financeiros, há para o associado, produtor rural com aversão ao risco, um maior valor marginal da perda de utilidade pelo provável insucesso econômico que irá incentivar a participação na governança corporativa da cooperativa.

Dessa forma, pode-se esperar que a participação social nos processos de decisão ocorra também em função do desempenho econômico em decorrência: a) do crescimento econômico que induz um maior custo de oportunidade de participação para os associados em conseqüência do crescimento do número de membros e da dispersão geográfica; b) do maior/menor nível de oferta de serviços e benefícios imediatos que poderá diminuir/aumentar necessidade de participação; e c) da freqüente aversão ao risco do produtor rural associado que induz a 
uma maior/menor participação, uma vez que o valor da perda/ganho de utilidade pelo insucesso/sucesso econômico é maior/menor que o custo de oportunidade de participação.

\section{O modelo}

Para explicar algumas evidências desse problema, é elaborado um modelo onde o pressuposto inicial é o de que, se a cooperativa tem um melhor desempenho econômico, há um aumento das externalidades econômicas e de serviços e, conseqüentemente, o cooperado terá um maior custo de oportunidade do que os benefícios da participação social e, portanto, não encontrará incentivo para construir um compromisso relacional por meio da participação social.

Nesse caso, os benefícios de preços e serviços aliados à utilidade e ao bem-estar criado pela cooperativa são suficientes, logo os custos de participação podem ser mais altos proporcionalmente do que os benefícios diferenciais criados em conseqüência da participação.

A dimensão de simples usuário apresenta somente benefícios, enquanto a construção de um compromisso relacional apresenta custos de participação. Dessa forma, o cooperado prefere a dimensão de usuário e não participa das atividades obrigatórias da cooperativa, como assembléias e comitês, entre outros.

Mas, à medida que a organização cooperativa apresenta um fraco desempenho econômico, o associado tem apenas duas opções: ou se desliga preventivamente do quadro social da cooperativa, se encontrar alternativa para os seus negócios, ou prefere participar com mais intensidade e assim aplicar seu tempo não somente para obter informações, como também de forma a criar acesso a serviços e benefícios nesse momento mais escassos e, deste modo, há um incentivo para a participação do associado.

Essa lógica pode explicar as correlações entre os desempenhos econômicos das cooperativas e a intensidade da participação social do associado, tanto na assembléia geral ordinária como em comitês educativos.

A participação também pode ser uma função da atividade econômica do associado na cooperativa, pode ocorrer devido à intensidade de assistência técnica por meio de agrônomos, zootecnistas e veterinários, pois esses são responsáveis pelas relações diretas entre a organização e 
seus associados nas áreas rurais, e podem, portanto, incentivar a participação e os compromissos relacionais.

Outra função importante para a participação pode ser a proporção dos membros associados na cooperativa entre todos os produtores rurais regionais. Essa característica mostra como a cooperativa é localmente importante e também pode indicar a distância entre o associado e a organização cooperativa.

Uma importante função é a proporção da produção agropecuária regional entregue na cooperativa. Se esta proporção é alta, o poder de mercado da cooperativa também é alto e pode influenciar na participação do seu associado. Quanto maior o poder de mercado, menores as necessidades para participação. Assim sendo, pode-se propor o seguinte modelo:

\section{Participação (assembléia geral e/ou o comitê educacional) do cooperado, em função da (atividade econômica, proporção de assistência técnica, proporção de membros entre os produtores rurais regionais, proporção de produtos agrícolas entregues $e$ desempenho econômico-financeiro).}

Espera-se um sinal positivo de correlação entre a participação e a atividade econômica do associado, a presença de assistência técnica, a proporção de membros entre os produtores rurais da região, e uma correlação negativa com o desempenho econômico-financeiro, isto é, melhor desempenho financeiro. Espera-se uma menor necessidade de participação.

\section{Análise de regressão}

Foram elaboradas duas análises com dados provenientes do Projeto de Políticas Públicas de Monitoramento da Autogestão de Cooperativas Agropecuárias $^{1}$ e efetuadas com informações do Sistema de Autogestão e Monitoramento da Organização das Cooperativas do Estado do Paraná (OCEPAR). Esse é o sistema de coleta de informações de maior experiência no monitoramento das cooperativas agroindustriais.

\footnotetext{
${ }^{1}$ Projeto de pesquisa coordenado pelo autor e desenvolvido em conjunto com o sistema cooperativista com o apoio institucional da FAPESP no âmbito dos projetos de políticas públicas.
} 
Para a análise, somente foram consideradas as cooperativas singulares, excluindo todas as cooperativas centrais, como também somente foram consideradas as cooperativas que apresentaram uma série total de dados, isto é, uma série coerente para todas as variáveis escolhidas. Nesta série de dados, 16 cooperativas apresentaram dados coerentes em todas as variáveis escolhidas e, portanto, participaram da análise cross-section para dados do ano de 1999. Essas cooperativas agrícolas, no entanto, representam as cooperativas agrícolas mais importantes do Estado do Paraná.

O modelo foi construído levando-se em consideração as relações logarítmicas de proporções, de forma a propiciar a normalização das variáveis, bem como isolar o efeito de tamanho das cooperativas sobre os números considerados. Por outro lado, após a eliminação da influência do tamanho, pelas proporções, pondera-se a regressão pelo número de associados de forma a possibilitar uma maior relevância para as cooperativas que apresentam um número maior de associados. Os modelos de regressão utilizados foram:

$$
\begin{aligned}
\text { Lpassago }= & \mathrm{a}+\mathrm{b} 1 \text { Lpasscmt }+\mathrm{b} 2 \text { Lpasstv }+ \text { b3 Lptecass } \\
& +\mathrm{b} 4 \text { Lpasspdt }+ \text { b5 Lptpdac }+ \text { b6 Bknt }+\mathrm{e}
\end{aligned}
$$

e:

$$
\begin{aligned}
\text { Lpasscmt }= & a+b 1 \text { Lpassatv }+ \text { b2 Lptecass }+ \text { b3 Lpasspdt } \\
& + \text { b4 Lptpdac }+ \text { b5 Bknt }+e
\end{aligned}
$$

As variáveis dependentes são o logaritmo da proporção de participação do associado em assembléia geral ordinária da cooperativa - Lpassago - e o logaritmo da proporção de participação do associado em comitê educativo - Lpasscmt. As variáveis independentes são:

a) Lpassatv que representa o logaritmo da proporção do número de associados ativos economicamente com a cooperativa;

b) Lptecass que representa o logaritmo da proporção de agrônomos, ou assistência técnica oferecida pela cooperativa aos produtores;

c) Lpasspdt é o logaritmo da proporção de associados no número de produtores rurais totais na área de atuação da cooperativa; 
d) Lptpdac representa o logaritmo da proporção da produção agrícola regional entregue na cooperativa;

e) Bknt é o desempenho econômico-financeiro da cooperativa representado pelo índice financeiro de Kanitz, e definida como variável binária.

Quanto ao índice de Kanitz ${ }^{2}$, se este for alto, a cooperativa tem uma situação financeira melhor e a variável assume valor 1; quando este índice é baixo, a cooperativa tem uma pior situação financeira, e esta variável assume valor 0 . Os resultados foram obtidos utilizando-se o programa estatístico SPSS e estão representados nas Tabelas 1 e 2 .

Optou-se pela seleção de variáveis efetuadas por meio do $B a$ ckward Elimination Method. Esse processo permite conhecer não somente o modelo originalmente proposto, mas também uma seqüência lógica de eliminação de variáveis e de ajustes, de forma a se obter um modelo o mais descritivo possível. Neste caso, inicia-se com todas as variáveis, e é removida, para os próximos passos, a variável cujo coeficiente tem menor significância - teste F - ou, em outras palavras, de menor capacidade de explicação. As matrizes de correlação e covariância mostram que não ocorreram problemas de multicolinearidade.

Na primeira regressão, para explicar a variável dependente de participação em Assembléias Gerais Ordinárias (AGOs), o resultado inicialmente não esperado é que a participação em comitês educativos em nível da comunidade não foi uma variável cujo coeficiente apresentasse um valor significativo para explicar a participação nas AGOs como era anteriormente esperado.

2 Índice de Kanitz é um índice financeiro obtido e ponderado de variáveis que representam a liquidez, o endividamento e a eficiência financeira das cooperativas agropecuárias. Para detalhes, consultar Marion (1991). 
.Tabela 1. Modelo de regressão, equação 1, em que o logaritmo da proporção da participação do associado em assembléia geral é a variável dependente e logaritmo da proporção da participação em comitês, da atividade econômica do associado, da proporção de assistência técnica por cooperado, da proporção de cooperados no total de produtores no local, da proporção da produção agrícola entregue, e o índice financeiro de Kanitz são

variáveis independentes, ponderadas pelo número de associados para

16 cooperativas agrícolas no Estado do Paraná em 1999.

\begin{tabular}{|c|c|c|c|c|c|}
\hline \multirow{2}{*}{\multicolumn{2}{|c|}{ Modelo }} & \multicolumn{2}{|c|}{ Coeficientes } & \multirow{2}{*}{$\mathbf{T}$} & \multirow{2}{*}{ Sig. } \\
\hline & & B & Erro & & \\
\hline \multirow{7}{*}{1} & (Constante) & $-4,271$ & 4,652 &,- 918 & 382 \\
\hline & LPASSATV &,- 977 & 1,878 &,- 520 & ,615 \\
\hline & LPTPDAC & $-1,421$ &, 674 & $-2,108$ & 064 \\
\hline & LPASSPDT & 1,447 &, 563 & 2,572 & ,030 \\
\hline & LPTECASS &,- 387 & 930 &,- 416 & 687 \\
\hline & BKNT &,- 615 & ,464 & $-1,324$ & 218 \\
\hline & LPASSCMT &,- 301 & ,302 &,- 999 & ,344 \\
\hline \multirow{6}{*}{2} & (Constante) & $-2,392$ & 1,067 & $-2,241$ & ,049 \\
\hline & LPASSATV &,- 797 & 1,751 &,- 456 & ,658 \\
\hline & LPTPDAC & $-1,543$ &, 581 & $-2,656$ & ,024 \\
\hline & LPASSPDT & 1,422 &, 536 & 2,653 & 024 \\
\hline & BKNT &,- 623 & 444 & $-1,401$ & 191 \\
\hline & LPASSCMT &,- 259 & ,272 &,- 952 & ,363 \\
\hline \multirow{5}{*}{3} & (Constante) & $-2,337$ & 1,021 & $-2,288$ & ,043 \\
\hline & LPTPDAC & $-1,700$ & ,452 & $-3,762$ & ,003 \\
\hline & LPASSPDT & 1,442 &, 514 & 2,803 & 017 \\
\hline & BKNT &,- 545 & ,395 & $-1,379$ & , 195 \\
\hline & LPASSCMT &,- 203 & ,233 &,- 869 & ,404 \\
\hline \multirow{4}{*}{4} & (Constante) & $-1,613$ & ,585 & $-2,759$ & ,017 \\
\hline & LPTPDAC & $-1,615$ & ,436 & $-3,699$ & ,003 \\
\hline & LPASSPDT & 1,558 & ,491 & 3,170 & ,008 \\
\hline & BKNT &,- 548 & ,391 & $-1,401$ &, 187 \\
\hline \multirow{3}{*}{5} & (Constante) & $-2,152$ & ,456 & $-4,714$ & ,000 \\
\hline & LPTPDAC & $-1,776$ & ,436 & $-4,071$ & ,001 \\
\hline & LPASSPDT & 1,635 & ,506 & 3,229 & ,007 \\
\hline
\end{tabular}

\begin{tabular}{cccc}
\hline Modelo & $\mathrm{R}$ & R Quadrado & R Quadrado ajustado \\
\hline $\mathbf{1}$ &, $813(\mathrm{a})$ &, 661 &, 435 \\
\hline $\mathbf{2}$ &, $809(\mathrm{~b})$ &, 655 &, 482 \\
\hline $\mathbf{3}$ &, $805(\mathrm{c})$ &, 647 &, 519 \\
\hline $\mathbf{4}$ &, $789(\mathrm{~d})$ &, 623 &, 529 \\
\hline $\mathbf{5}$ &, $749(\mathrm{e})$ &, 562 &, 494 \\
\hline
\end{tabular}

Fonte: dados de pesquisa - tabela gerada pelo programa estatístico SPSS 
Tabela 2. Modelo de regressão, equação 2, em que o logaritmo da proporção da participação do associado em comitês educativos é a variável dependente e o logaritmo da proporção da atividade econômica do associado, da proporção de assistência técnica por cooperado, da proporção de cooperados no total de produtores no local, da proporção da produção agrícola entregue, e o índice financeiro de Kanitz são variáveis independentes, ponderadas pelo número de associados para 16 cooperativas agrícolas no Estado do Paraná - em 1999.

\begin{tabular}{|c|c|c|c|c|c|}
\hline \multirow{2}{*}{\multicolumn{2}{|c|}{ Modelo }} & \multicolumn{2}{|c|}{ Coeficientes } & \multirow[b]{2}{*}{$\mathbf{t}$} & \multirow{2}{*}{ Sig. } \\
\hline & & B & Erro & & \\
\hline \multirow{6}{*}{1} & (Constante) & $-7,771$ & 4,212 & $-1,845$ &, 095 \\
\hline & LPASSATV & $-3,074$ & 1,712 & $-1,796$ & , 103 \\
\hline & LPTPDAC & ,543 & ,685 & ,792 & ,447 \\
\hline & LPASSPDT &,- 400 & ,576 &,- 694 &, 503 \\
\hline & LPTECASS & $-1,047$ & ,917 & $-1,142$ & ,280 \\
\hline & BKNT &,- 220 & ,482 &,- 457 &, 658 \\
\hline \multirow{5}{*}{2} & (Constante) & $-8,191$ & 3,960 & $-2,069$ &, 063 \\
\hline & LPASSATV & $-2,808$ & 1,551 & $-1,811$ & ,098 \\
\hline & LPTPDAC & ,437 & ,621 & ,703 & ,497 \\
\hline & LPASSPDT &,- 372 &, 552 &,- 674 &, 514 \\
\hline & LPTECASS & $-1,087$ &, 879 & $-1,237$ &, 242 \\
\hline \multirow{4}{*}{3} & (Constante) & $-8,446$ & 3,851 & $-2,193$ & ,049 \\
\hline & LPASSATV & $-2,916$ & 1,507 & $-1,934$ &, 077 \\
\hline & LPTPDAC & ,260 &, 550 & ,472 & ,646 \\
\hline & LPTECASS & $-1,200$ & ,844 & $-1,422$ &, 180 \\
\hline \multirow{3}{*}{4} & (Constante) & $-7,475$ & 3,156 & $-2,369$ &, 034 \\
\hline & LPASSATV & $-2,458$ & 1,119 & $-2,196$ &, 047 \\
\hline & LPTECASS &,- 964 & ,658 & $-1,464$ &, 167 \\
\hline \multirow{2}{*}{5} & (Constante) & $-2,865$ & ,194 & $-14,736$ &, 000 \\
\hline & LPASSATV & $-3,208$ & 1,035 & $-3,099$ &, 008 \\
\hline
\end{tabular}

\begin{tabular}{|c|c|c|c|}
\hline Modelo & $\mathbf{R}$ & R Quadrado & R Quadrado Ajustado \\
\hline 1 &, $728(a)$ &, 530 & ,295 \\
\hline 2 & ,721(b) &, 520 & ,345 \\
\hline 3 &, $707(\mathrm{c})$ &, 500 & ,375 \\
\hline 4 & ,701(d) & ,491 & ,412 \\
\hline 5 & ,638(e) & ,407 & 364 \\
\hline
\end{tabular}

Fonte: dados de pesquisa - tabela gerada pelo programa estatístico SPSS 
O mesmo ocorreu com o comportamento dos coeficientes da variável de proporção de associados ativos, em que se esperava que este apresentasse uma correlação positiva e significativa, e de proporção de assistência técnica, em que também se esperava a mesma correlação positiva. Isto é, tinha-se a expectativa de que maior número de membros ativos economicamente e um maior número de técnicos, prestando assistência em campo, pudesse originar uma maior participação nas AGOs, mas isso não ocorreu.

Nessa análise estatística, o modelo 4 apresentou uma maior capacidade de explicação, R quadrado ajustado de 0,529. Nesse modelo, pode-se observar que o coeficiente da variável de proporção de produção regional entregue na cooperativa apresentou um coeficiente significativo, mas negativo, assim, se cresce o poder de mercado da cooperativa, decresce a presença em AGOs, talvez por não haver alternativas de mercado para o associado na destinação da sua produção e, desta forma, serem muito altos os custos de oportunidade de participar nas estruturas de decisão e investir tempo nas estruturas de governança.

Por outro lado, a variável de proporção de associados entre o total de produtores da área de ação apresentou um coeficiente significativo e de correlação positiva para com a participação em AGOs. Isso pode ser interpretado como a concentração de associação, ou intensidade de associação, favorecendo a participação. Talvez, também, porque a proximidade local favoreça a participação.

Por último, deve-se observar que a variável binária do índice de Kanitz apresentou um coeficiente negativo e significativo, o que, nesse caso, corrobora a hipótese apresentada de uma relação inversamente proporcional entre as variáveis, isto é, em uma situação de piora neste índice, por conseguinte, na situação financeira da cooperativa, há um estímulo à participação.

Quanto à proporção de participação em comitês educativos em nível das comunidades, os resultados são diferentes. O modelo de melhor explicação foi o modelo 4, apresentando um $\mathrm{R}$ quadrado ajustado de 0,412 , e em que as variáveis de proporção de associados ativos e de proporção de assistência técnica por associados apresentaram coeficientes significativos e de correlação inversamente proporcional. Assim, se há mais atividade, e maior presença da co- 
operativa por meio de sua assistência técnica, também haverá uma menor participação em comitês.

Portanto, aparentemente, os comitês educativos têm a função de suprir deficiência na relação com os associados em função da pequena presença da cooperativa em campo, por meio de assistência técnica, engenheiros agrônomos e veterinários.

Também nesse nível de participação, aparentemente, a condição de desempenho financeiro da cooperativa, aqui mensurada pela variável binária do índice de Kanitz, não apresentou coeficiente significativamente diferente de zero.

\section{Considerações finais}

Tem-se para essas considerações finais dois fatos. O primeiro: que as organizações cooperativas apresentam no Brasil uma estrutura de direitos de propriedade pouco definida, o que pode ser considerado como uma limitação institucional; e o segundo: que freqüentemente as cooperativas no Brasil apresentam a função objetivo de oferecer melhores preços, serviços imediatos e benefícios aos membros, sem priorizar uma distribuição futura de resultados econômicos em dinheiro - distribuição de sobras.

Quanto às considerações a respeito do número de sócios para a participação em assembléias gerais, obteve-se, analisando graficamente os dados, uma relação de tendência logarítmica que mostra que, quanto maior é a cooperativa em número de sócios, menor é a proporção de participação, e assim, em conseqüência do maior número de sócios, menor será o poder proporcional de decisão de cada cooperado e maiores serão os custos de oportunidade de participação.

Deve-se considerar que a organização cooperativa deverá apresentar benefícios e serviços perceptíveis ao cooperado para que este tenha incentivo econômico para transacionar com a cooperativa e participar. $\mathrm{O}$ acesso diferenciado a informações, serviços e benefícios da cooperativa poderá, em conseqüência de uma estrutura de direitos de propriedade muito pouco definida, ser um incentivo para a participação.

Considera-se que a participação também ocorra em cooperativas agropecuárias com o objetivo de gerar e de intensificar relações informais, e que o incentivo para tanto são as vantagens obtidas por meio do 
exercício de influência, que poderá ser mais intensa em função do tamanho da cooperativa e dos conseqüentes problemas de monitoramento.

Os modelos de regressão permitem considerar que há uma relação inversamente proporcional entre o desempenho econômico e a participação em AGOs e, assim, parte das considerações pode ser julgada verdadeira. Logo, uma discussão plausível é que, quanto maior é o sucesso econômico da cooperativa, essa poderá oferecer uma maior quantidade de serviços e benefícios disponíveis; desta maneira, maior seriam também os custos de oportunidade de participação e, portanto, não haveria incentivos para a participação social.

Na participação em comitês educativos, que ocorrem em nível da comunidade, os resultados permitem uma discussão diferente, pois como há relações negativas com a proporção de assistência técnica e a proporção de associados ativos, isso permite considerar que estes comitês possivelmente existam para intensificar a participação e as relações sociais em organizações que apresentem possivelmente problemas de atividade do quadro associado.

Poder-se-ia considerar que esta situação estaria relacionada com uma provável estratégia de reforçar as relações sociais, na forma de comitês educativos com o objetivo de solucionar uma situação de baixa participação e atividade dos cooperados. A variável independente de proporção de cooperados ativos economicamente apresenta uma relação inversamente proporcional com a variável dependente proporção de participação em comitês educativos.

Desta forma, é inicialmente correta e corroborável a hipótese de Meister (SOARES, 1984) de que, conforme o crescimento econômico da organização cooperativa, a lógica do capital suplanta a social e a organização cooperativa passa a operar cada vez mais exclusivamente de acordo com uma lógica apenas de eficiência econômica e do mercado, se afastando cada vez mais das questões de relações sociais, e assim cada vez mais importante será a consideração dos custos de oportunidade do associado para a participação social.

Esses resultados também permitem considerar que as proposições de que a maior participação dos associados em cooperativas é condição para o melhor desempenho da organização são verdadeiras, contudo podem não ocorrer em realidade, o que é problemático, visto que a partici- 
pação nas AGOs está negativamente correlacionada com o desempenho econômico, e a participação em comitês educativos está também negativamente correlacionada com o número de cooperados ativos economicamente. Assim, essa situação pode causar conseqüências para a transparência da gestão e o bom desempenho da governança corporativa.

Por fim, pode-se indicar, ainda, uma agenda de pesquisa na qual a participação em empreendimentos coletivos, como as cooperativas, é de fundamental importância para gerar transparência de governança corporativa, havendo uma dicotomia a ser discutida, pois, quanto melhor for o desempenho econômico das cooperativas, maior serão os custos de oportunidade de participação e menor será a participação e, portanto, menos transparente será o processo de gestão e governança, exigindo custos adicionais de comunicação para suplantar essa deficiência.

\section{Referências bibliográficas}

BIALOSKORSKI NETO, S. Gobierno y papel de los cuadros directivos en las cooperativas brasileñas: estudio comparativo. Revista de Economía Pública Social y Cooperativa, Valencia, n. 48, abr. 2004a.

Economia das organizações cooperativas: uma análise da influência da cultura e das instituições. Ribeirão Preto, 2004. Tese (Livre Docência) - Faculdade de Economia, Administração e Contabilidade de Ribeirão Preto da Universidade de São Paulo, 2004b. $178 f$.

BIRCHALL, J.; SIMMONS, R. What motivates members to participate? A reworking of a theoretical model and some findings. In: KARAFOLAS, S; SPEAR, R; STRYJAN, Y. Local society \& global economy: the role of co-operatives. Naoussa: Hellin, 2002. cap. 2, p.131-157.

COOK, M.; ILIOPOULOS, C. Solutions to property rights constraints in producer-owned and controlled organizations: prerequisite for agrichain leadership? In: INTERNATIONAL CONFERENCE ON CHAIN MANAGEMENT IN AGRIBUSINESS AND FOOD INDUSTRY, 3., 1998, Wageningen. Proceedings... Wageningen: Wageningen Agricultural University, 1998, p. 541-553.

ESCHENBURG, R. Una breve introducción a la teoría económica de la cooperación._Perspectiva Econômica, São Leopoldo, v. 13, p. 7-14, 1983. 
GRANOVETTER, M. Economic action and social structure: the problem of embeddedness. The American Journal of Sociology, Chicago, v 91, p. 481-510, 1985.

HENDRIKSE, G. W. J. Contingent controls rights and loss aversion in agricultural cooperatives. IN: EUROPEAN RESEARCH CONFERENCE ON VERTICAL MARKETS AND COOPERATIVE HIERARCHIES: THE ROLE OF COOPERATIVES IN THE INTERNATIONAL AGRI-FOOD INDUSTRY, 2003, Bad Herrenalb. Proceedings... Bad Herrenalb, 2003. 10 p. Disponível em: < http://www.flec.kvl.dk/kok/coop03/ > . Acesso em: set. 2003.

MARION, J.C. Contabilidade empresarial. São Paulo: Atlas, 1991. $540 \mathrm{p}$.

OLSON, M. The logic of collective action: public goods and the theory of groups. Cambridge: Harvard University Press, 1965. 186 p.

PINHO, D. B. O cooperativismo no Brasil. São Paulo: Saraiva, 2003. $357 \mathrm{p}$.

ROEMER, J. E. Egalitarian perspectives: essays in philosophical economics. Cambridge: Cambridge University Press, 1996. 356 p.

SINGER, P.; SOUZA, A.R. A economia solidária no Brasil. São Paulo: Contexto, 2000. 360p.

SOARES, R.P. Sociologia da cooperação: o dilema Meister-Zupanov. Perspectiva Econômica. São Leopoldo, n. 45, p. 91-100, 1984.

Recebido em novembro de 2005 e revisto em julho de 2006. 